\title{
Oral care with chlorhexidine seems effective for reducing the incidence of ventilator-associated pneumonia
}

\author{
Abstracted from \\ Hua F, Xie H, Worthington HV, Furness S, Zhang Q, Li C. \\ Oral hygiene care for critically ill patients to prevent ventilator-associated pneumonia. \\ Cochrane Database Syst Rev 2016; 10: Art.No.:CD008367.DOI:10.1002/14651858.CD008367.pub3. \\ Address for correspondence: Luisa Fernandez Mauleffinch, Managing Editor, Cochrane Oral Health Group, School of Dentistry, The University of \\ Manchester, JR Moore Building, Oxford Road, Manchester, M1 3 9PL, UK. \\ E-mail: luisa.fernandez@manchester.ac.uk
}

\section{Question: Does good oral hygiene reduce the incidence of ventilator-associated pneumonia?}

Data sources Electronic databases searched were Cochrane Oral Health's Trials Register, the Cochrane Central Register of Controlled Trials (CENTRAL), Medline Ovid, Embassy Ovid, LILACS BIREME Virtual Health Library, CINAHL EBSCO, Chinese Biomedical Literature Database, China National Knowledge Infrastructure, Wan Fang Database and VIP Database ClinicalTrials.gov and the World Health Organisation International Clinical Trials Registry Platform for ongoing trials. No restrictions on language or date of publication.

Study selection Randomised controlled trials (RCTs) were included evaluating $\mathrm{OHC}$ in the form of mouthwashes, swabs or toothbrushing or in combination in critically ill patients receiving mechanical ventilation.

Data extraction and synthesis Two reviewers carried out data extraction independently. Study authors were contacted for additional information. Random-effects meta-analyses were performed where data could be pooled.

Results Thirty-eight RCTs (6,016 participants) were included. Five trials (13\%) were assessed at low risk of bias, 26 studies (68\%) high and seven studies (18\%) of unclear risk of bias. There were four main comparisons; chlorhexidine ( $\mathrm{CHX}$ mouthrinse or gel) versus placebo/ usual care, toothbrushing versus no toothbrushing, powered versus manual toothbrushing and comparisons of oral care solutions.

Evidence from 18 RCTs (2451 participants, 86\% adults) shows that $\mathrm{CHX}$ mouthrinse or gel, as part of $\mathrm{OHC}$, reduces the risk of VAP compared to placebo or usual care from $25 \%$ to about $19 \%$ (RR 0.74 , $95 \%$ confidence intervals $(\mathrm{Cl}) 0.61$ to $0.89, \mathrm{P}=0.002$, heterogeneity $\mathrm{I} 2$ $=31 \%$ ). Number needed to treat (NNT) $=17$ ( $95 \% \mathrm{Cl} 10$ to 33).

There is no evidence of a difference between $\mathrm{CHX}$ and placebo/ usual care for the outcomes of mortality (RR 1.09, 95\% Cl 0.96 to $1.23, \mathrm{P}=0.18, \mathrm{I} 2=0 \%, 15 \mathrm{RCTs}, 2163$ participants, moderate quality evidence), duration of mechanical ventilation (MD -0.09 days, $95 \%$ $\mathrm{Cl}-1.73$ to 1.55 days, $\mathrm{P}=0.91, \mathrm{I} 2=36 \%$, five RCTs, 800 participants,

This paper is based on a Cochrane Review published in the Cochrane Library 2016, issue 10 (see www.thecochranelibrary. com for information). Cochrane Reviews are regularly updated as new evidence emerges and in response to feedback, and the Cochrane Library should be consulted for the most recent version of the review. low quality evidence) or duration of intensive care unit (ICU) stay (MD 0.21 days, $95 \% \mathrm{Cl}-1.48$ to 1.89 days, $\mathrm{P}=0.81, \mathrm{I} 2=9 \%$, six $\mathrm{RCTs}, 833$ participants, moderate quality evidence). There is insufficient evidence to determine the effect of $\mathrm{CHX}$ on duration of systemic antibiotics, oral health indices, caregivers' preferences or cost. Only two studies reported any adverse effects, and these were mild with similar frequency in $\mathrm{CHX}$ and control groups.

The effect of toothbrushing ( \pm antiseptics) is uncertain on the outcomes of VAP (RR $0.69,95 \% \mathrm{Cl} 0.44$ to $1.09, \mathrm{P}=0.11,12=$ $64 \%$, five RCTs, 889 participants, very low quality evidence) and mortality (RR $0.87,95 \% \mathrm{Cl} 0.70$ to $1.09, \mathrm{P}=0.24, \mathrm{I} 2=0 \%$, five RCTs, 889 participants, low quality evidence) compared to $\mathrm{OHC}$ without toothbrushing ( \pm antiseptics).

There is insufficient evidence to determine whether toothbrushing affects duration of mechanical ventilation, duration of ICU stay, use of systemic antibiotics, oral health indices, adverse effects, caregivers' preferences or cost.

Only one trial (78 participants) compared use of a powered toothbrush with a manual toothbrush, providing insufficient evidence to determine the effect on any of the outcomes of this review.

Fifteen trials compared various other oral care solutions. There is very weak evidence that povidone iodine mouthrinse is more effective than saline/placebo (RR $0.69,95 \% \mathrm{Cl} 0.50$ to $0.95, \mathrm{P}=0.02,12=74 \%$, three studies, 356 participants, high risk of bias) and that saline rinse is more effective than saline swab (RR $0.47,95 \% \mathrm{Cl} 0.37$ to $0.62, \mathrm{P}<0.001,12$ $=84 \%$, four studies, 488 participants, high risk of bias) in reducing VAP. Due to variation in comparisons and outcomes among trials, there is insufficient evidence concerning the effects of other oral care solutions. Conclusions The results from high quality evidence found that oral hygiene care $(\mathrm{OHC})$, including chlorhexidine mouthwash or gel, reduces the risk of developing ventilator-associated pneumonia in critically ill patients from $25 \%$ to about $19 \%$. However, there is no evidence of a difference in the outcomes of mortality, duration of mechanical ventilation or duration of ICU stay.

There is no evidence that $\mathrm{OHC}$ including both antiseptics and toothbrushing is different from $\mathrm{OHC}$ with antiseptics alone, and some weak evidence to suggest that povidone iodine mouthrinse is more effective than saline/placebo, and saline rinse is more effective than saline swab in reducing VAP. There is insufficient evidence to determine whether powered toothbrushing or other oral care solutions are effective in reducing VAP. There is also insufficient evidence to determine whether any of the interventions evaluated in the studies are associated with adverse effects. 


\section{Commentary}

Nosocomial infections are recognised as an important cause of mortality and morbidity in patients with prolonged stays in hospital, and have a detrimental impact in critically ill patients. Despite many suggested strategies for prevention, the prevalence of nosocomial infections remains elevated. Ventilator associated pneumonia (VAP) is the most common infection in intubated patients. ${ }^{1}$

Diverse reasons put patients at higher risk, such as the presence of the endotracheal tube, body position, possible aspiration of contaminated secretions from the oropharynx or gastrointestinal tract and an open and dry mouth have all been linked to VAP. The direct association with pulmonary infections and oral health has been explored in different studies. ${ }^{2}$

A series of strategies or bundle strategies are suggested for the prevention of VAP.

Most of the VAP bundle care include strategies such as hand hygiene, personal protective equipment, environmental cleaning, cleaning of equipment, bed position avoiding a supine position, subglottic secretion drainage and oral care. ${ }^{3,4}$

There is an agreement within all the recommendations that oral health is an important risk factor to control in order to prevent VAP. However, there is a lack of consensus regarding the technique of application, frequency and choice of antimicrobial concentration, particularly for chlorhexidine since solutions vary in concentration $(0.12 \%$ up to $2 \%) .{ }^{5}$ Some of those concentrations are not available in all countries.

The updated version with results of the well conducted Cochrane review agrees with several systematic reviews addressing the same topic. One particular review, completed by Price et al. and published in 2014, raised the concern of the safety of chlorhexidine and influenced the final recommendation for chlorhexidine use by some organisations. The network metaanalysis suggests that both selective digestive decontamination (SDD) and selective oropharyngeal decontamination (SOD) are superior to chlorhexidine use. The effect of chlorhexidine on mortality was considered by the authors, even though mortality was not the primary outcome in any of the studies and mortality was seen in only one of the 11 included studies. The recommendations are uncertain due to the heterogeneity among the studies. Most of the US and the world recommendations include the use of chlorhexidine in different concentrations in the bundle of preventions. $6,7,8,9,10$

Chlorhexidine, it seems, can also be effective perioperatively in decreasing the incidence of nosocomial infections and postoperative pneumonia, as results from a systematic review suggest. ${ }^{11}$

It makes sense that good oral hygiene, simple in technique, can have a positive effect on minimising the development of VAP. Despite no mention of the dosage, interval of use or lack of outcomes such as mortality, duration of mechanical ventilation or duration of ICU stay not being assessed, VAP incidence is still reduced and that alone is an important outcome.
Since there is a lack of agreement regarding the type of oral care protocols that achieve the best results, a randomised clinical trial was conducted to fill the gap, specifically with chlorhexidine.

The study used two different techniques, chlorhexidine $0.2 \%$ swabbing and toothbrush with chlorhexidine gluconate $0.2 \%$. In addition, the suction catheter was used three times a day. ${ }^{12}$ The results of the study show that both techniques were no different in reducing VAP. It seems the number of days on the ventilator had an impact on results, however, oral care is a key factor for prevention.

A different randomised clinical trial recently published concluded that $2 \%$ is better than $0.2 \%$. The side-effects reported were mild and reversible mucosal irritation. ${ }^{5}$

There are other systematic reviews also demonstrating the reduction in VAP in critically ill patients by the regular use of chlorhexidine, after intubation and mechanical ventilation. ${ }^{13,14,15}$

It is obvious that oral healthcare intervention alone will not prevent VAP, since it is multifactorial in nature. As a result, the patient will receive the greatest benefit if prevention is performed as a bundle as is recommended; oral care with chlorhexidine seems a good intervention.

\section{Analia Veitz-Keenan and Debra M Ferraiolo NYU College of Dentistry, New York, USA}

1. Tokmaji G, Vermeulen H, Müller M C A, Kwakman P H, Schultz M J, Zaat S A. Silver-coated endotracheal tubes for prevention of ventilator-associated pneumonia in critically ill patients. Cochrane Database Syst Rev 2015; 8: CD009201. DOI:10.1002/14651858.CD009201.pub.

2. Segers P, Speekenbrink R G, Ubbink D T, van Ogtrop M L, de Mol B A. Prevention of nosocomial infection in cardiac surgery by decontamination of the nasopharynx and oropharynx with chlorhexidine gluconate: a randomized clinical trial. JAMA 2006; 296: $2460-2466$.

3. Pássaro L, Harbarth S, Landelle C. Prevention of hospital-acquired pneumonia in non ventilated adult patients: a narrative review. Antimicrob Resist Infect Control 2016; 5: 43.

4. Klompas M, Branson R, Eichenwald E C et al. Strategies to prevent ventilatorassociated pneumonia in acute care hospitals: 2014 update. Infect Control Hosp Epidemiol 2014; 35: S133-S154.

5. Zand $F$, Zahed L,Mansouri P et al. The effects of oral rinse with $0.2 \%$ and $2 \%$ chlorhexidine on oropharyngeal colonization and ventilator associated pneumonia in adults' intensive care units. / Crit Care 2017; 40: 318-322.

6. https://www.cdc.gov/hai/vap/vap.html (accessed August 2017).

7. https://www.hpsc.ie/az/microbiologyantimicrobialresistance/infectioncontrolandhai/ guidelines/File,12530,en.pdf (accessed August 2017).

8. http://www.sicsag.scot.nhs.uk/hai/care.html (accessed August 2017).

9. https://www.nhmrc.gov.au/book/australian-guidelines-prevention-and-controlinfection-healthcare-2010/b4-2-3-ventilation (accessed August 2017).

10. http://www.ihi.org/resources/Pages/Tools/HowtoGuidePreventVAP.aspx (accessed August 2017).

11. Spreadborough P, Lort S, Pasquali S et al.; Preventing Postoperative Pneumonia Study Group and the West Midlands Research Collaborative. A systematic review and metaanalysis of perioperative oral decontamination in patients undergoing major elective surgery. Perioper Med (Lond) 2016; 5: 6. DOI: 10.1186/s13741-016-0030-7.

12. Chacko R, Rajan A, Lionel P, Thilagavathi M, Yadav B, Premkumar J. Oral decontamination techniques and ventilator associated pneumonia. Br J Nurs 2017 26: 594-599. DOI: 10.12968/bjon.2017.26.11.594.

13. Chan E Y, Ruest A, Meade M O, Cook D J. Oral decontamination for prevention of pneumonia in mechanically ventilated adults: systematic review and meta-analysis. BMI 2007; 334: 889.

14. Labeau S O, Van de Vyver K, Brusselaers N, Vogelaers D, Blot SI. Prevention of ventilator-associated pneumonia with oral antiseptics: a systematic review and metaanalysis. Lancet Infect Dis 2011; 11: 845-854.

15. Villar C C, Pannuti C M, Nery D M, Morillo C M, Carmona M I, Romito G A Effectiveness of Intraoral Chlorhexidine Protocols in the Prevention of VentilatorAssociated Pneumonia: Meta-Analysis and Systematic Review. Respir Care 2016; 61: $1245-1259$.

Evidence-Based Dentistry (2017) 18, 113-114. doi:10.1038/sj.ebd.6401272 